SUBJECT AREAS:

RNA PROBES

FLUORESCENCE IMAGING

Received

31 July 2013

Accepted

2 December 2013

Published

9 January 2014

Correspondence and requests for materials should be addressed to R.H.S. (robert.singer@ einstein.yu.edu)

\section{Background free imaging of single mRNAs in live cells using split fluorescent proteins}

Bin $\mathrm{Wu}^{1,2}$, Jiahao Chen ${ }^{1}$ \& Robert H. Singer ${ }^{1,2}$

'Department of Anatomy and Structural Biology, Albert Einstein College of Medicine of Yeshiva University, ${ }^{2}$ Gruss Lipper Biophotonic Center, Albert Einstein College of Medicine of Yeshiva University.

We describe a technique for imaging single mRNAs in living cells based on fluorescent protein (FP) complementation. We employ the high affinity interaction between the bacterial phage MS2/PP7 coat proteins and their respective RNA binding motifs as an RNA scaffold to bring two halves of a split-FP together to image single reporter mRNAs without background fluorescence.

. he application of genetically encoded fluorescent tags allows imaging mRNA dynamics in live cells or transgenic organisms. The MS2 system, exploiting the high affinity interaction between the MS2 coat protein (MCP) and MS2 binding site (MBS), has been widely used to fluorescently label mRNA in various model organisms ${ }^{1}$. However, a limitation of this approach is that it requires a level of MCP in excess of the mRNA in order to bind, thereby resulting in a constant background ${ }^{2}$. This can be troublesome for visualizing mRNA, particularly in deep tissue or whole body imaging. A solution to this is to employ protein complementation ${ }^{3}$, used to study protein-mRNA interactions $s^{4}$ or label mRNAs ${ }^{5-7}$. We report an application of this approach, whereby a fluorescent protein (FP) is split into two non-fluorescent fragments, which are fused to two different RNA binding proteins (RBP). When the RBPs bind to their corresponding motifs that are positioned adjacent to each other in the RNA, the two complementation fragments are brought close to each other and reconstitute a complete mature FP. A previous implementation of this approach used RBP either from a endogenous eukaryotic protein (eIF4A) (therefore could not be used in eukaryotic cells $)^{5}$, or had low binding affinity $\left(\mathrm{K}_{\mathrm{d}}>90 \mathrm{nM}\right.$, and therefore required a high concentration of protein relative to the mRNAs, increasing background and obviating single molecule sensitivity $)^{6}$. Recently we have engineered another RNA-binding protein and its cognate RNA motifs in a similar manner as the MS2 system. PP7 bacteriophage coat protein (PCP) binds to its own PP7 binding site (PBS) with high affinity $\left(\mathrm{K}_{\mathrm{d}}=1.6 \mathrm{nM}\right)$ but only weakly interacts with the MBS $\left(\mathrm{K}_{\mathrm{d}}>1 \mu \mathrm{M}\right)^{8}$. Therefore the RBPs, MS2 and PP7 can be used to image a distinct population of mRNAs in the same cells ${ }^{9}$.

\section{Results}

We combined MS2 and PP7 (PBS and PCP analogous to the MBS and MCP) to form a fluorescence complementation labeling system. MCP and PCP were fused to the $\mathrm{N}$ - or $\mathrm{C}$ - fragments of the FP Venus respectively (Fig. A, B). To increase coexpression efficiency, MCP-VenusN and PCP-VenusC were combined into a polycistronic plasmid using an internal ribosome entry site (IRES) (Fig. B). The MBS and PBS were fused together, amplified into a tandem array (12xMBS-PBS) and inserted into the $3^{\prime}$ untranslated region (3'UTR) of the reporter mRNA (Fig. B). Twelve repeats were employed to facilitate single molecule detection of mRNAs since up to 24 fluorescent proteins can reconstitute on the mRNA. The coat proteins and the reporter mRNA were cotransfected into U2OS cells. The reporter mRNAs could then be imaged with single molecule sensitivity (Fig. C-E and supplementary movie). The coat proteins, MCP and PCP both contained a nuclear localization signal (NLS) so that only when they are bound to the mRNA are they visible in the cytoplasm. Therefore there was no fluorescent signal in the nucleus indicating the absence of fluorescence from free coat protein, unlike the situation with a single RBP-FP where the NLS concentrates the unbound protein there(Fig. F). Tracking of fluorescent particles labeled with the BiFC construct shows identical diffusion behavior as the ones labeled with 24xMBS (Fig. G) ${ }^{10}$. Since it has been well documented that MS2 labeled fluorescent particles are single molecules of $\mathrm{mRNA}^{2,11}$, this demonstrates that the BiFC fluorescent particles are consistent with single mRNAs. If the 
(A) without RNA

With RNA

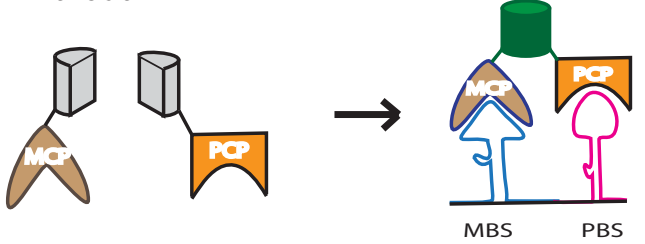

(B)
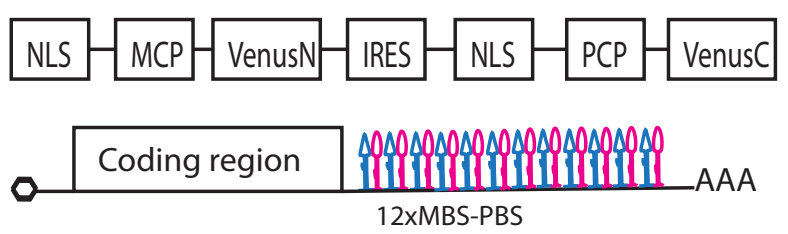

(C)

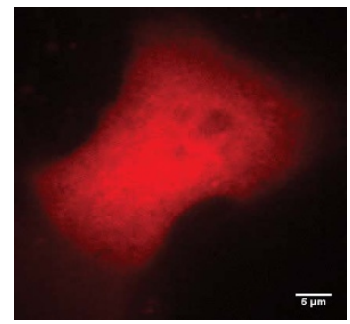

(F)

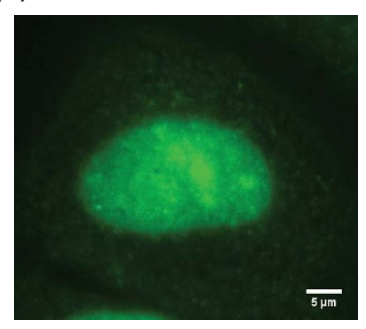

(D)

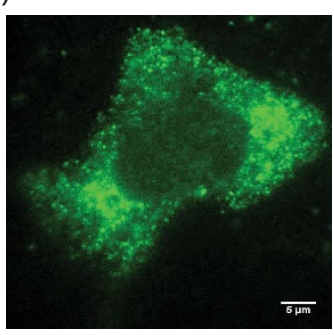

(E)
(G)

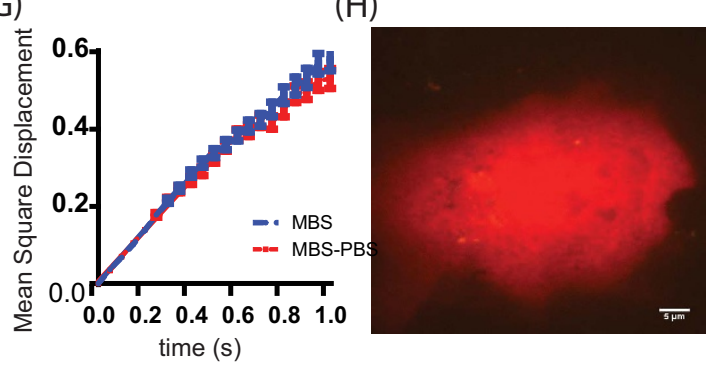

Figure | (A) Schematic of fluorescence complementation using the MS2 and PP7 systems. The two fragments of an FP, fused to MCP and PCP respectively, are brought together by adjacent binding sites and form a complete FP. (B) Schematic of the split-FP coat proteins and the reporter mRNA (NLS: nuclear localization signal; IRES: internal ribosome entry site; VenusN: the N-terminal fragment of Venus; VenusC: the C-terminal fragment of Venus. (C-E) The reporter mRNA, encoding a far-red fluorescent protein and with 12xMBS-PBS inserted in the $3^{\prime}$ UTR, was transiently transfected with the split-FP coat protein construct in U2OS cells: (C) the far-red channel; (D) the mRNA channel; (E) the merge. For a streaming acquisition of the green channel, see the online supplementary movie. (F) A reporter mRNA eqFP670-24xMS2, labeled with NLS-tdMCP-EGFP, was imaged in the green channel. Although mRNA was visible as labeled spots in the cytoplasm, the nucleus contained free unbound coat proteins and was bright. (G) The reporter mRNAs labeled with 12xMBS-PBS ( 5 cells, 935 tracks) or 24xMS2 (7 cells, 1427 tracks) are tracked in live cells. The mean square displacements were plotted as a function of time. The slope determines the diffusion constants of mRNA, $24 x M B S: 0.17 \mu \mathrm{m}^{2} / \mathrm{s}, 12 \mathrm{xMBS}-\mathrm{PBS}: 0.16 \mu \mathrm{m}^{2} / \mathrm{s}$, which are identical within experimental uncertainty. (H) When the reporter mRNA eqFP670-24xMS2 was cotransfected with the split-FP coat proteins, only the red channel was visible; the mRNA was not labeled in the green channel since PCP-VenusC did not bind to mRNA and form complementation with MCP-VenusN.

reporter mRNA contained only the MBS, but lacked an adjacent PBS, the $\mathrm{BiFC}$ construct did not yield discrete fluorescence particle (Fig. H), as expected.

\section{Discussion}

We successfully demonstrated the application of the high affinity MS2 and PP7 systems in labeling mRNA without background. This reagent therefore does not add fluorescent free coat proteins to the system and hence provides a very sensitive means of detecting single mRNAs. This technology will be of great practical value for imaging mRNAs in tissues or in whole animal, where the free coat protein confounds the image by adding an accumulated background through tissue depth.

$\mathrm{BiFC}$ approach has a few limitations that need careful handling. First, the fluorescent protein fragments can self-associate at very high concentrations independent of interaction, and consequently contribute to a diffusive background ${ }^{12}$. To eliminate this background, coat proteins must be expressed at a low level. Second, the complemented fluorescent protein formation is irreversible and the reconstituted fluorophore needs time for maturation ${ }^{12}$. Hence this approach is best suited to study long-lived mRNAs and not appropriate to image real time transcription. Nuclear RNA events are not visible because the mRNA is exported before it becomes fluorescent. This may be a useful feature by which to detect mRNAs that are nuclear-sequestered, such as long, non-coding RNAs.

\section{Methods}

To construct MBS-PBS hybrid sites, oligonucleotides containing MBS and PBS were digested and self-ligated in the presence of digesting enzymes. The DNA corresponding to $3 \mathrm{xMBS}-\mathrm{PBS}$ was gel purified and inserted into a pcr4 plasmid backbone (Invitrogen). The plasmid was digested and another $3 \times$ MBS-PBS was inserted to generate pcr4-6xMBS-PBS. The same procedure was repeated to generate pcr4$12 \mathrm{xMBS}-\mathrm{PBS}$. 
The construction of plasmids, transfection and microscopy are detailed in the supplementary information.

1. Bertrand, E. et al. Localization of ASH1 mRNA particles in living yeast. Mol Cell 2, 437-445 (1998).

2. Wu, B., Chao, J. A. \& Singer, R. H. Fluorescence fluctuation spectroscopy enables quantitative imaging of single mRNAs in living cells. Biophys J 102, 2936-2944 (2012).

3. Hu, C. D., Chinenov, Y. \& Kerppola, T. K. Visualization of interactions among bZIP and Rel family proteins in living cells using bimolecular fluorescence complementation. Mol Cell 9, 789-798 (2002).

4. Rackham, O. \& Brown, C. M. Visualization of RNA-protein interactions in living cells: FMRP and IMP1 interact on mRNAs. EMBO J 23, 3346-3355, doi:10.1038/ sj.emboj.7600341 (2004).

5. Valencia-Burton, M., McCullough, R. M., Cantor, C. R. \& Broude, N. E. RNA visualization in live bacterial cells using fluorescent protein complementation. Nat Methods 4, 421-427 (2007).

6. Ozawa, T., Natori, Y., Sato, M. \& Umezawa, Y. Imaging dynamics of endogenous mitochondrial RNA in single living cells. Nat Methods 4, 413-419 (2007).

7. Yiu, H.-W., Demidov, V. V., Toran, P., Cantor, C. R. \& Broude, N. E. RNA Detection in Live Bacterial Cells Using Fluorescent Protein Complementation Triggered by Interaction of Two RNA Aptamers with Two RNA-Binding Peptides. Pharmaceuticals 4, 494-508 (2011).

8. Chao, J. A., Patskovsky, Y., Almo, S. C. \& Singer, R. H. Structural basis for the coevolution of a viral RNA-protein complex. Nat Struct Mol Biol 15, 103-105 (2008).

9. Hocine, S., Raymond, P., Zenklusen, D., Chao, J. A. \& Singer, R. H. Singlemolecule analysis of gene expression using two-color RNA labeling in live yeast. Nat Methods 10, 119-121 (2013).

10. Jaqaman, K. et al. Robust single-particle tracking in live-cell time-lapse sequences. Nat Methods 5, 695-702 (2008).
11. Fusco, D. et al. Single mRNA molecules demonstrate probabilistic movement in living mammalian cells. Curr Biol 13, 161-167 (2003).

12. Kerppola, T. K. Design and implementation of bimolecular fluorescence complementation (BiFC) assays for the visualization of protein interactions in living cells. Nat Protoc 1, 1278-1286 (2006).

\section{Acknowledgments}

This work was supported by the grant GM84364 and EB 013571 from National Institutes of Health to R.H.S. The plasmid eqFP670 was a kind gift from Dr. Vladislav Verkusha, Albert Einstein College of Medicine. The authors thank Xiuhua Meng and Jefferey A. Chao for cloning.

\section{Author contributions}

B.W. and R.H.S. designed the research. B.W. and J.C. performed the experiments and analyzed the data. B.W. and R.H.S. wrote the paper.

\section{Additional information}

Supplementary information accompanies this paper at http://www.nature.com/ scientificreports

Competing financial interests: The authors declare no competing financial interests. How to cite this article: Wu, B., Chen, J.H. \& Singer, R.H. Background free imaging of single mRNAs in live cells using split fluorescent proteins. Sci. Rep. 4, 3615; DOI:10.1038/ srep03615 (2014).

(c) (i) $(-$ This work is licensed under a Creative Commons Attribution-

BY Nc No NonCommercial-NoDerivs 3.0 Unported license. To view a copy of this license, visit http://creativecommons.org/licenses/by-nc-nd/3.0 